\title{
Revised photometric distances to nearby dwarf galaxies in the IC 342/Maffei complex
}

\author{
I. Karachentsev ${ }^{1}$, I. Drozdovsky ${ }^{2}$, S. Kajsin ${ }^{1}$, L.O. Takalo ${ }^{3}$, P. Heinämäki ${ }^{3}$, and M. Valtonen ${ }^{3}$ \\ 1 Special Astrophysical Observatory, N.Arkhyz, Stavropolski Kraj 357147, Russia \\ 2 Astronomical Institute, St.-Petersburg State University, Petrodvoretz 198904, Russia \\ 3 Tuorla Observatory, Turku, Finland
}

Received November 12; accepted December 16, 1996

\begin{abstract}
The results of DAOPHOT photometry of several hundred stars in five irregular galaxies are presented using $V$ and $I$ CCD frames, obtained with the Nordic Optical Telescope under a 0.6 arcsec seeing. Based on the brightest blue and red stars we estimate the following distances to the galaxies: $3.2 \mathrm{Mpc}$ for UGCA 105, 2.6 Mpc for UGCA 86, 1.8 Mpc for UGCA 92, $1.7 \mathrm{Mpc}$ for NGC 1569, and 1.7 Mpc for Cas 1. The problem of membership of the galaxies to the IC $342 /$ Maffei complex is briefly discussed ${ }^{1}$.
\end{abstract}

Key words: galaxies: irregular — galaxies: distances galaxies: stellar content

\section{Introduction}

At the intersection of the Local Supergalactic plane and the Milky Way zone and in the vicinity of the bright spiral IC 342 there are 12 galaxies with radial velocities below $500 \mathrm{~km} / \mathrm{s}$. Judging by the amplitude of internal motions, some of them (Maffei 1, Maffei 2, Dwingeloo 1) are massive galaxies, which have escaped notice until recently because of strong interstellar absorption. In addition to the galaxies known previously (NGC 1560, NGC 1569, UGCA 86, UGCA 92, UGCA 105) new objects have been found in the region: Cas $2=$ Dwingeloo 1 (Kraan-Korteweg et al. 1994), Cas 1 (Huchtmeier et al. 1995), MB 1 (McCall \& Buta 1995; Huchtmeier \& van Driel 1996), Dwingeloo 2 (Burton et al. 1996), Cam A (Karachentsev 1994), and Cam B (Huchtmeier et al. 1997). The surveys of the region, which have been undertaken with the Tautenburg 2-meter telescope plates

Send offprint requests to: L.O. Takalo

1 Tables 2 to 6 are only available in electronic form at the CDS via anonymous ftp to cdsarc.u-strasbg.fr (130.79.128.5) or via http://cdsweb.u-strasbg.fr/Abstract.html
(Börngen \& Karachentseva 1985) and also with POSSII films (Karachentseva \& Karachentsev 1997) have revealed some other candidates to be probable members of this nearby complex of galaxies. The distribution of the galaxies is presented in Fig. 1 in galactic coordinates. The galaxies with corrected radial velocities $V_{0}$ below $500 \mathrm{~km} / \mathrm{s}$ are indicated with open circles, and other possible new members of the complex are noted with crosses.

Except for Maffei 1, Dwingeloo 1, Dwingeloo 2, and MB 1, situated in the strongest absorption zone, the rest of the galaxies with $V_{0}<500 \mathrm{~km} / \mathrm{s}$ have already been resolved into stars. Based on the luminosity of the brightest blue and red stars the following distance have been estimated: $3.3 \mathrm{Mpc}$ for UGCA 105 (Tikhonov et al. 1992), $2.1 \mathrm{Mpc}$ for IC 342 and $1.9 \mathrm{Mpc}$ for UGCA 86 (Karachentsev \& Tikhonov 1993), 3.8 Mpc for NGC 1560 (Karachentsev et al. 1991), 1.8 Mpc for NGC 1569 and 2.2 Mpc for UGCA 92 (Karachentsev et al. 1994), $2.3 \mathrm{Mpc}$ for Maffei 2 (Tikhonov \& Karachentsev 1994), and $0.8 \mathrm{Mpc}$ for Cas 1 (Tikhonov 1996). The mean distance of the IC $342 /$ Maffei complex $\langle D\rangle=2.3 \mathrm{Mpc}$ derived from these data agrees well with the earlier estimates made by Spinrad et al. (1973) and McCall (1989), but disagrees significantly with the value $D=4.2 \pm 0.5 \mathrm{Mpc}$, obtained by Luppino \& Tonry (1993) for Maffei 1 via surface brightness fluctuation method.

The problem of the distance to the IC 342/Maffei $1+2$ group was discussed recently by Krismer et al. (1996). Estimating distance moduli to NGC 1560 and UGCA 105 by the Tully-Fisher method, these authors obtained a distance of $\langle D\rangle=(3.6 \pm 0.5) \mathrm{Mpc}$, and ascribed it to the rest of the galaxies in the group. However, this suggestion is not at all evident in the case of the scattered association of galaxies which is seen along the Local Supercluster plane.

It should be stressed that if it is sufficiently nearby the IC 342/Maffei $1+2$ group might affect the trajectories of galaxies in the Local Group (Zheng et al. 1991; Valtonen 
et al. 1993; Peebles 1994). Therefore, it is important to obtain precise distances to the galaxies in question.

\section{Observations and reductions}

The galaxies UGCA 86, UGCA 92, UGCA 105, NGC 1569, and Cas 1 were observed in February 5-7, 1995 at the Nordic 2.5-meter telescope in the $V$ and $I$ bands of the Kron-Cousins system. The observations were carried out with a TK 1024A CCD chip having 1024 by 1024 pixels each 24 microns in size. It provided a $3{ }^{\prime} .0 \times 3{ }^{\prime} .0$ field of view with the resolution of $0^{\prime \prime} 176$ per pixel. The CCD quantum efficiency is about $70 \%$ in each of the bands, and the readout noise is $6.5 \mathrm{e}$. The frames of the galaxies were obtained under quite good photometric conditions with a seeing of FMHM $=0 . \prime 5-0 .{ }^{\prime \prime} 7$. Table 1 describes the exposures.

After subtracting dark frame, flat-fielding and cleaning of cosmic ray events the photometric processing of the frames was performed with DAOPHOT and ALLSTAR packages (Stetson 1987). Equatorial photometric standards from Landolt (1992) were observed in order to transform instrumental magnitudes into the standard $V$ and $I$ system. According to our estimate the total error in the zero-point does not exceed 0.05 mag for any of the galaxies.

\section{The results of the photometry and the distance moduli}

Following the traditional approach developed by Sandage \& Tammann (1974) and de Vaucouleurs (1978), we use the brightest blue $(V-I<+0.5)$ and red $(V-I>+2.0)$ stars as distance indicators to the galaxies. The photometric distance modulus, $\mu_{0}(B)$, was derived via the mean apparent magnitude of three the brightest blue stars, $<B(3 B)>$, from the relation

$\mu_{0}(B)=1.51<B(3 B)>-0.51 B_{\mathrm{T}}-A_{B}+4.14$,

where $B_{\mathrm{T}}$ is the total apparent magnitude of the galaxy, and $A_{B}$ is the foreground galactic extinction. Relation (1) reflects well the known correlation between the luminosity of blue supergiants and the luminosity of their parent galaxy. Its numerical coefficients were calibrated by Piotto et al. (1992) and Karachentsev \& Tikhonov (1994) using galaxies with known distances from Cepheids. To derive $B$ magnitudes we use the empirical relation.

$B-V=0.80(V-I)$,

which is valid in general for Landolt's stars.

For deriving a modulus from red supergiants de Vaucouleurs (1978) recommended the use of their magnitude $\left\langle M_{v}(3 R)\right\rangle=-7^{\mathrm{m}} \cdot 7$, irrespective of the luminosity of the parent galaxy. We applied this rule to all five galaxies observed.

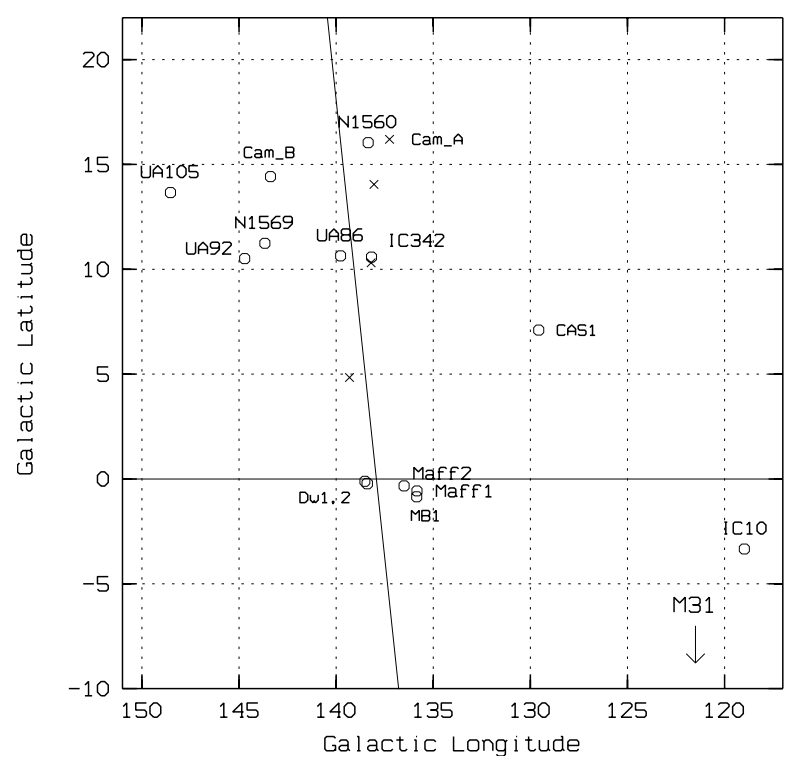

Fig. 1. The distribution of galaxies in the IC 342/Maffei complex in galactic coordinates. The open circles represent galaxies with radial velocities $V_{0}<500 \mathrm{~km} / \mathrm{s}$, the crosses correspond to other probable members of the complex. The inclined solid line is the Supergalactic equator

\section{1. $U G C A 105$}

According to RC 3 (de Vaucouleurs et al. 1991) this irregular galaxy of Sm type has a standard angular diameter of 5.5 arcmin. A reproduction of its central part in the $I$ band is presented in Fig. 2. Applying the ALLSTAR programme, we carried out the photometry of 568 stars seen on both CCD frames and having the image parameters: $|\mathrm{SHARP}|<2, \quad|\mathrm{CHI}|<2$, and $\sigma(V)<0.20 \mathrm{mag}$, where $\sigma(V)$ is a formal error of the $V$-magnitude. SHARP and $\mathrm{CHI}$ are measures of the starts shape and the goodness of fit, respectively, in the reductions. SHARP is a measure of the difference beetween the observed width of the object and the width of the psf model. The results of our photometry are given in Table 2. Its columns contain: (1) - the star number, (2) and (3) - the star coordinates expressed in pixels, (4) - the $V$ magnitude, (5) - the colour index $V-I,(6)$ - the image parameter, SHARP.

Figure 3 shows the "Colour-Magnitude" diagram derived from these data. The brightest stars with $V<20.5$ and "neutral" colour $0.7<V-I<2.2$ are evidently foreground stars. The population of the brightest blue stars of the galaxy itself appears in the region of $V>22.5$ and $V-I<0.6$ in good agreement with the data by Tikhonov et al. (1992). Among them we select stars \#\#459, 226, and 261 as the brightest blue supergiant candidates, excluding from the consideration two cases, \#\#20 and 449 which have not sharp stellar images. Their mean apparent 


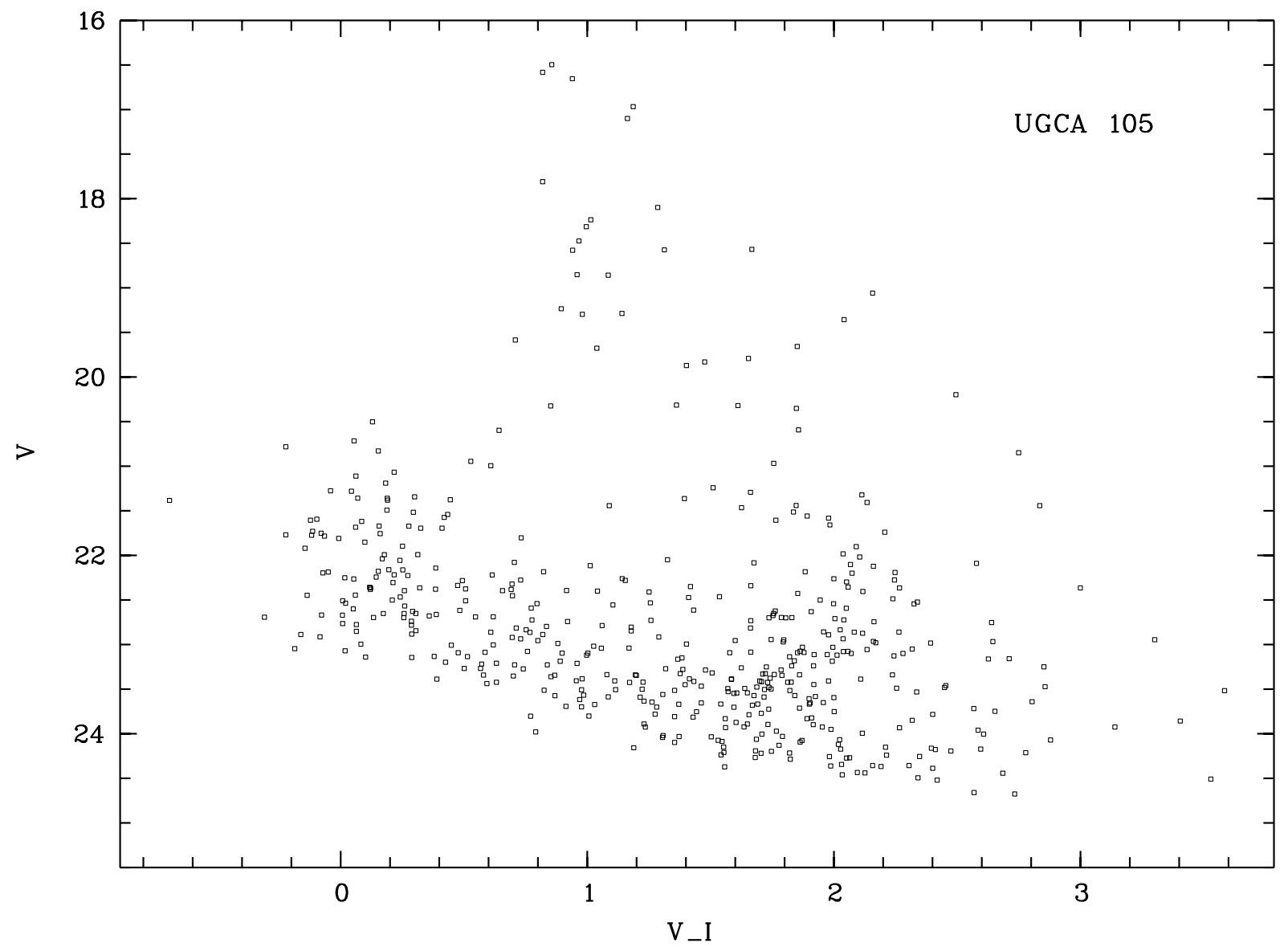

Fig. 3. Colour-Magnitude diagram for 568 stars in UGCA 105

obtained in the $I$ band. The total magnitude of UGCA 92 is rather controversial. The catalog RC3 gives an apparent magnitude of 13.8 , but in the catalog of Karachentseva \& Sharina (1987) its magnitude is $16^{\mathrm{m}} 4$. According to the CCD photometry by Karachentseva et al. (1996) the galaxy has $B_{\mathrm{T}}=15.22$ and $(B-V)_{\mathrm{T}}=1.34$. From present measurements after corrections for the Galactic stars the total magnitude and total colour of UGCA 92 are $V_{\mathrm{T}}=15 \cdot 42,(V-I)_{\mathrm{T}}=1.58$, or $B_{\mathrm{T}}=16^{\mathrm{m}} \cdot 15$ and $(B-V)_{\mathrm{T}}=1.26$.

The results of our photometry of 184 stars are presented in Table 3, which is structured in the same way as Table 2. Among them 100 stars, situated inside the visible boundary of the galaxy, are indicated by "1" in the last column. Figure 5 shows the Colour-Magnitude diagram based on these data. The red colour of many stars is caused obviously by the strong interstellar extinction. We estimated the absorption value, $A_{B}$, from the galaxy colour. Adopting for an irregular type galaxy the mean true colour, $\left\langle B-V>_{0}=0.36 \pm 0.02\right.$ (RC3), we obtain a colour excess of $E(B-V)=0.90 \pm 0.05$ or $A_{B}=$ $4.2 E(B-V)=3$ m.78. The brightest blue stars belonging to the galaxy itself are easily distinguished from the fore- ground stars. For the three brightest, \# 82, 57 and 117 we obtain from relation (2) the mean: $\langle B(3 B)\rangle=22.60$ and $\langle B-V\rangle=+0.98$, which gives the distance modulus of $\mu_{0}(B)=26^{\mathrm{m}} 25$ or $D=1.78 \mathrm{Mpc}$.

From the $\mathrm{C}-\mathrm{M}$ diagram it appears that the brightest red supergiants of the galaxy may have $V \simeq 23^{\mathrm{m}}$, $V-I \simeq 3.0$. However, to distinguish them from foreground stars is a difficult task. Comparing the present photometry of individual stars with our old CCD data (Karachentsev et al. 1994), we find a systematic difference, $\left\langle V_{\text {new }}-V_{\text {old }}>=+0.42 \pm 0.05\right.$, the reason of which remains unclear to us.

\section{3. $U G C A \quad 86=V I I Z w 9$}

The dwarf galaxy UGCA 86 with its radial velocity $V_{\mathrm{h}}=$ $+67 \mathrm{~km} / \mathrm{s}$ and standard angular diameter $a_{25}=4.5$ remains still the only indisputable satellite of the spiral IC 342. Figure 6 reproduces a CCD frame in the $I$ band, covering the central part of UGCA 86 and also its bright southern concentration (= VIIZw9). According to Huchtmeier \& Richter (1986) the total magnitude of 


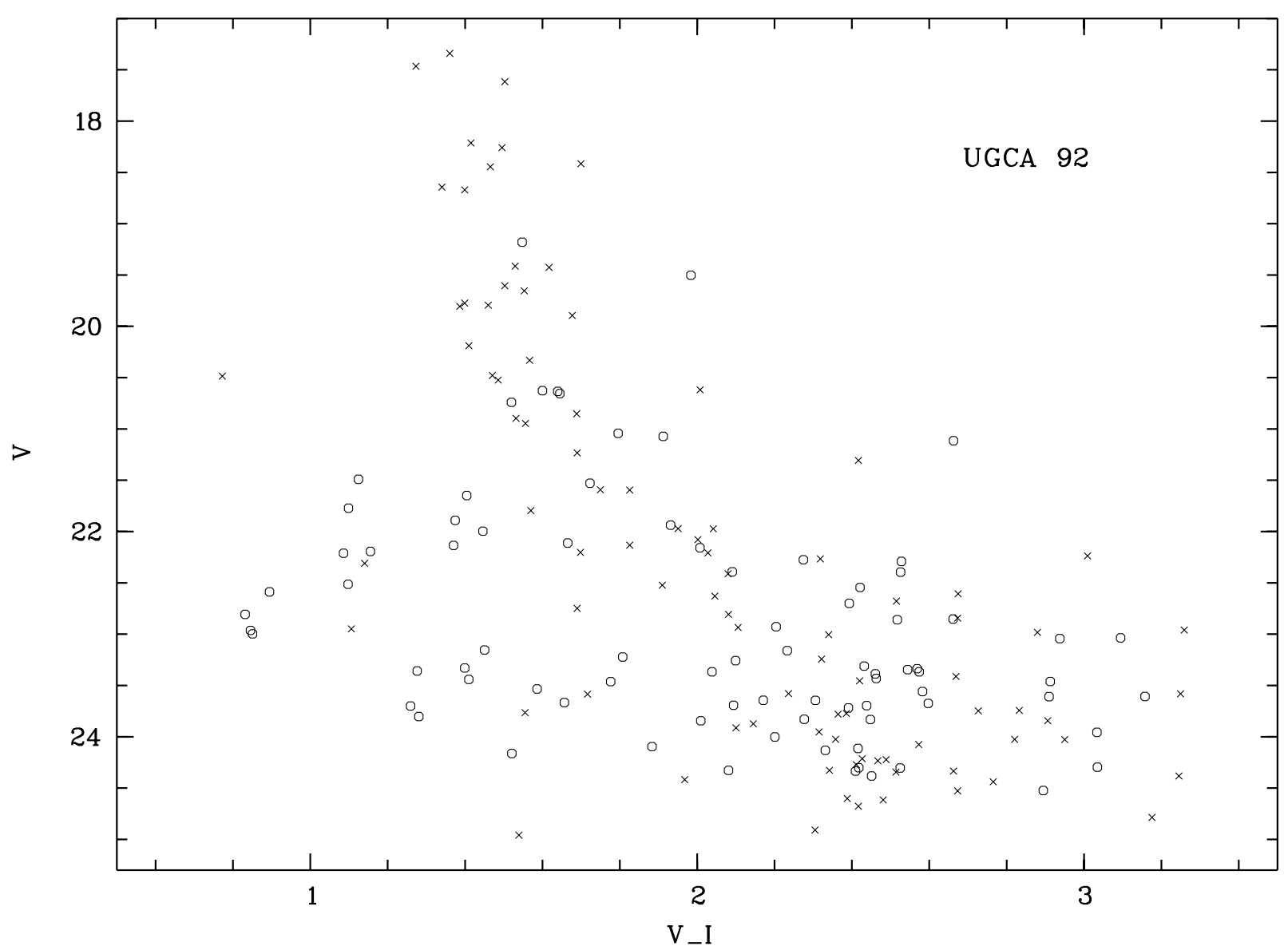

Fig. 5. Colour-Magnitude diagram for UGCA 92. the stars inside and outside the galaxy body are indicated by open circles and crosses, respectively

Table 1. Journal of observations

\begin{tabular}{|ccrc|}
\hline Object & Filter & Exposure & Date \\
\hline UGCA 86 & $V$ & $600 \mathrm{~s}$ & Feb. 6, 1995 \\
& $I$ & $600 \mathrm{~s}$ & \\
\hline UGCA 92 & $V$ & $600 \mathrm{~s}$ & Feb. 6, 1995 \\
& $I$ & $600 \mathrm{~s}$ & \\
\hline UGCA 105 & $V$ & $600 \mathrm{~s}$ & Feb. 6, 1995 \\
& $I$ & $600 \mathrm{~s}$ & \\
\hline NGC 1569 & $V$ & $300 \mathrm{~s}$ & Feb. 7, 1995 \\
& $I$ & $300 \mathrm{~s}$ & \\
& $R$ & $60 \mathrm{~s}$ & \\
\hline Cas 1 & $V$ & $600 \mathrm{~s}$ & Feb. 5, 1995 \\
& $I$ & $600 \mathrm{~s}$ & \\
\hline
\end{tabular}

supergiant candidates we obtain a modulus of $\mu_{0}(R)=$ 26.97. The mean of the two estimates, $27^{\mathrm{m}} 12 \pm 0{ }^{\mathrm{m}} 15$, corresponds to a distance of $D=2.65 \mathrm{Mpc}$, which exceeds appreciably the old estimate, $1.86 \mathrm{Mpc}$, by Karachentsev \& Tikhonov (1993). Both these estimates agree quite well with the mean distance of IC $342(D=2.3 \mathrm{Mpc})$. The difference between the new and the old photometric zeropoints is negligible, $+0{ }^{\mathrm{m}} 08 \pm 0.06$, but the increase of the distance estimate is rather caused by a new selection of blue and red stars for the supergiants of the galaxy.

\section{4. $N G C 1569=U G C 3056=\operatorname{Arp} 210=V I I Z w 16$}

This peculiar galaxy with $V_{\mathrm{h}}=-89 \mathrm{~km} / \mathrm{s}$ has been studied by many authors (Ables 1971; Arp \& Sandage 1985; Karachentsev et al. 1994; Krismer et al. 1995). The high brightness gradient across NGC 1569 and a presence of dusty furrows in its body make stellar photometry very hard. The galaxy image in the $I$ band is shown in Fig. 8. Its upper part reproduces one half of the original frame, and the lower one shows the same part of the galaxy after subtracting a frame smoothed with a window of $10 \times(\mathrm{FWHM})$, allowing a filtering of extended emission or unresolved stars. An application of the ALLSTAR package allowed us to perform the photometry of more than 500 stars inside the galaxy as well as arround it. However, a lot of stars, especially within its central part, were excluded due to the condition $|\mathrm{SHARP}|>2$. The results of 


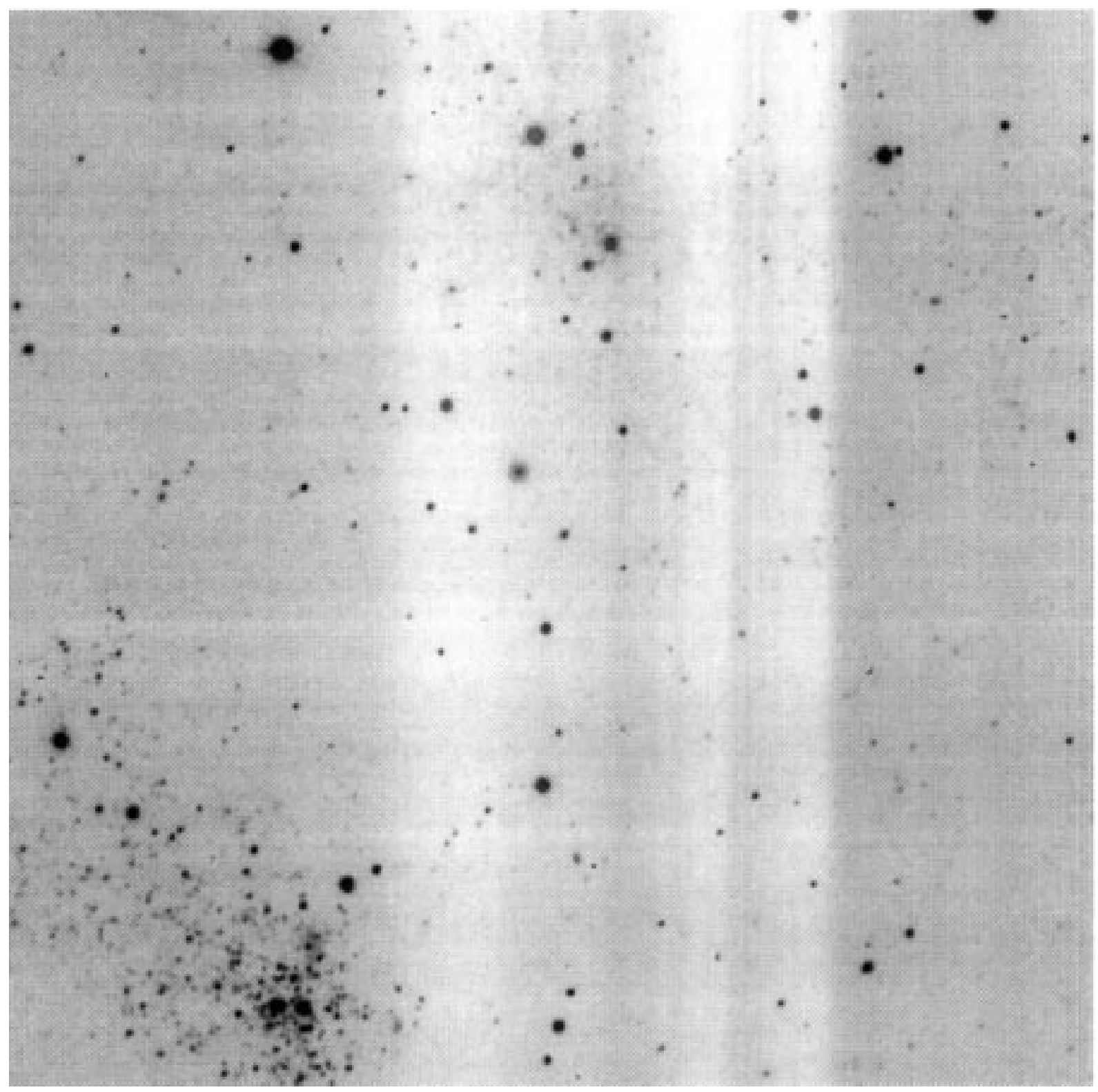

Fig. 6. The central part of UGCA 86 (above) and the bright southern superassociation (the lower left corner) in the $I$ band

the photometry for the remainding 173 stars are given in Table 5, which contains also $R$ magnitudes measured from a short exposure frame. Foreground stars, situated on the opposite (southern) half of the CCD frame, are indicated by the number "1". The distribution of stars in the plane $V$ vs. $V-I$ is shown in Fig. 9. The stars from the northern and southern halfs of the CCD frame are noted by open circles and crosses, respectively. In this diagram we plot also 113 stars from the central part of NGC 1569, measured with the Hubble Space Telescope (O'Connel et al. 1994). These data fit each other quite well.

In contrast to the galaxies considered previously, NGC 1569 has no sharp left "shoulder" in its C-M diagram, caused by a population of blue supergiants. Because of this, a distance modulus of the galaxy cannot be derived accurately.

Adopting the stars \#18, 50 and 70 as the three brightest blue supergiant candidates, we obtain for them $\langle V(3 B)\rangle=19.85$ and $\langle V-I\rangle=+0.25$ or $<B(3 B)>=20$ m 05 . Using the total magnitude of the galaxy, $B_{\mathrm{T}}=11^{\mathrm{m}} 86$, and an extinction of $A_{B}=2 \cdot{ }^{\mathrm{m}} 18$ (RC3) this magnitude gives a modulus of $\mu_{0}(B)=26.19$ in a good agreement with the estimate of $\langle\mu\rangle=26.27$ by Karachentsev et al. (1994). It should be noted, however, that we did not find suitable red supergiant candidates based on their colour $(V-I>2.6)$ and magnitude $\left(V \simeq 20^{\mathrm{m}}\right)$. In comparison with the results of aperture photometry (Karachentsev et al. 1994), the ALLSTAR 
Table 7. Some global properties of the galaxies

\begin{tabular}{|c|c|c|c|c|c|c|c|c|c|}
\hline Name & $\begin{array}{l}V_{\mathrm{h}} \\
V_{0}\end{array}$ & $\begin{array}{l}a_{25} \\
b / a\end{array}$ & $\begin{array}{c}B_{\mathrm{T}} \\
\text { Type }\end{array}$ & $\begin{array}{c}A_{B} \\
b^{\circ}\end{array}$ & $\begin{array}{c}W_{50} \\
\log S(\mathrm{HI})\end{array}$ & $\begin{array}{l}\mu_{0} \\
D\end{array}$ & $\begin{array}{l}A_{25} \\
M_{B}\end{array}$ & $\begin{array}{c}M_{25} / L \\
M_{\mathrm{HI}} / M_{25}\end{array}$ & Notes \\
\hline UGCA 92 & $\begin{array}{l}-99 \\
+89\end{array}$ & $\begin{array}{l}2.0 \\
.48\end{array}$ & $\begin{array}{l}16.15 \\
\mathrm{Im}\end{array}$ & $\begin{array}{l}3.78 \\
10.5\end{array}$ & $\begin{array}{c}61 \\
3.44\end{array}$ & $\begin{array}{c}26.25 \\
1.78\end{array}$ & $\begin{array}{c}2.0 \\
-14.3\end{array}$ & $\begin{array}{c}3.7 \\
0.17\end{array}$ & $\begin{array}{l}\text { present } \\
\text { paper }\end{array}$ \\
\hline NGC 1569 & $\begin{array}{c}-89 \\
+102\end{array}$ & $\begin{array}{l}3.6 \\
.49\end{array}$ & $\begin{array}{c}11.86 \\
\mathrm{Ip}\end{array}$ & $\begin{array}{l}2.18 \\
11.2\end{array}$ & $\begin{array}{c}74 \\
3.67\end{array}$ & $\begin{array}{c}26.19 \\
1.73\end{array}$ & $\begin{array}{c}2.5 \\
-16.9\end{array}$ & $\begin{array}{c}0.6 \\
0.15\end{array}$ & $\begin{array}{l}\text { present } \\
\text { paper }\end{array}$ \\
\hline NGC 1560 & $\begin{array}{c}-36 \\
+170\end{array}$ & $\begin{array}{l}9.8 \\
.15\end{array}$ & $\begin{array}{c}12.16 \\
\mathrm{Sd}\end{array}$ & $\begin{array}{l}1.13 \\
16.0\end{array}$ & $\begin{array}{l}125 \\
4.14\end{array}$ & $\begin{array}{c}27.37 \\
2.98\end{array}$ & $\begin{array}{c}7.7 \\
-17.3\end{array}$ & $\begin{array}{c}2.8 \\
0.19\end{array}$ & $\begin{array}{l}\text { (KTGBS, 91) } \\
1)\end{array}$ \\
\hline Maffei 2 & $\begin{array}{c}-2 \\
+226\end{array}$ & $\begin{array}{l}3.8: \\
.49\end{array}$ & $\begin{array}{l}16.0 \\
\mathrm{SBb}\end{array}$ & $\begin{array}{c}8.2 \\
-0.3\end{array}$ & $\begin{array}{l}305 \\
4.18\end{array}$ & $\begin{array}{c}26.77 \\
2.26\end{array}$ & $\begin{array}{c}11.9 \\
-19.4\end{array}$ & $\begin{array}{c}5.2 \\
0.01\end{array}$ & $(\mathrm{TK}, 94)$ \\
\hline IC 342 & $\begin{array}{l}+33 \\
+247\end{array}$ & $\begin{array}{c}20.9 \\
.95\end{array}$ & $\begin{array}{l}9.1 \\
\text { Scd }\end{array}$ & $\begin{array}{l}2.56 \\
10.6\end{array}$ & $\begin{array}{l}151 \\
5.34\end{array}$ & $\begin{array}{c}27.29 \\
2.87\end{array}$ & $\begin{array}{c}29.7 \\
-20.8\end{array}$ & $\begin{array}{c}\text { 1.9: } \\
0.17 \text { : }\end{array}$ & $\begin{array}{l}(\mathrm{KT}, 93) \\
2)\end{array}$ \\
\hline Cas 1 & $\begin{array}{l}+35 \\
+283\end{array}$ & $\begin{array}{l}2.2 \\
.77\end{array}$ & $\begin{array}{c}16.38 \\
\mathrm{Im}\end{array}$ & $\begin{array}{c}5.50 \\
7.1\end{array}$ & $\begin{array}{c}49 \\
3.31\end{array}$ & $\begin{array}{c}26.15 \\
1.70\end{array}$ & $\begin{array}{c}3.1 \\
-15.3\end{array}$ & $\begin{array}{c}2.8 \\
0.06\end{array}$ & $\begin{array}{l}\text { present } \\
\text { paper }\end{array}$ \\
\hline UGCA 86 & $\begin{array}{c}+67 \\
+275\end{array}$ & $\begin{array}{l}4.5 \\
.68\end{array}$ & $\begin{array}{l}14.2 \\
\mathrm{Sm}\end{array}$ & $\begin{array}{l}3.82 \\
10.6\end{array}$ & $\begin{array}{c}99 \\
4.41\end{array}$ & $\begin{array}{c}27.12 \\
2.65\end{array}$ & $\begin{array}{c}7.1 \\
-17.0\end{array}$ & $\begin{array}{c}4.2 \\
0.26\end{array}$ & $\begin{array}{l}\text { present } \\
\text { paper }\end{array}$ \\
\hline Cam B & $\begin{array}{l}+75 \\
+265\end{array}$ & $\begin{array}{l}2.2 \\
.50\end{array}$ & $\begin{array}{c}16.1 \\
\operatorname{Im}\end{array}$ & $\begin{array}{l}1.5: \\
14.4\end{array}$ & $\begin{array}{c}20 \\
2.20\end{array}$ & $\begin{array}{c}- \\
(3.0)\end{array}$ & $\begin{array}{c}1.9 \\
-13.2\end{array}$ & $\begin{array}{c}0.9 \\
0.32\end{array}$ & (HKK, 97) \\
\hline Dwing 2 & $\begin{array}{c}+94 \\
+314\end{array}$ & $\begin{array}{l}1.0: \\
.36\end{array}$ & $\begin{array}{l}\text { 20.5: } \\
\text { Im }\end{array}$ & $\begin{array}{c}9: \\
-0.2\end{array}$ & $\begin{array}{l}100 \\
3.13\end{array}$ & $\begin{array}{c}- \\
(4.2)\end{array}$ & $\begin{array}{c}6.2 \\
-16.9\end{array}$ & $\begin{array}{c}3.4 \\
0.05\end{array}$ & $(\mathrm{BVKH}, 96)$ \\
\hline Dwing 1 & $\begin{array}{l}+110 \\
+330\end{array}$ & $\begin{array}{l}2.0 \\
.63\end{array}$ & $\begin{array}{l}19: \\
\mathrm{SBb}\end{array}$ & $\begin{array}{c}10: \\
-0.1\end{array}$ & $\begin{array}{l}188 \\
3.95\end{array}$ & $\begin{array}{c}- \\
(4.4)\end{array}$ & $\begin{array}{c}16.1 \\
-19.6\end{array}$ & $\begin{array}{c}2.8 \\
0.03\end{array}$ & $\begin{array}{l}\text { (BVKH, 96) } \\
(\text { HLSSW, 95) }\end{array}$ \\
\hline UGCA 105 & $\begin{array}{l}+111 \\
+279\end{array}$ & $\begin{array}{l}5.5 \\
.62\end{array}$ & $\begin{array}{c}13.24 \\
\mathrm{Sm}\end{array}$ & $\begin{array}{l}1.48 \\
13.7\end{array}$ & $\begin{array}{l}118 \\
3.97\end{array}$ & $\begin{array}{c}27.54 \\
3.22\end{array}$ & $\begin{array}{c}6.3 \\
-16.0\end{array}$ & $\begin{array}{l}11.0 \\
0.12\end{array}$ & $\begin{array}{l}\text { present } \\
\text { paper }\end{array}$ \\
\hline MB 1 & $\begin{array}{l}+189 \\
+420\end{array}$ & $\begin{array}{l}2.4 \\
.42:\end{array}$ & $\begin{array}{c}\text { 20.5: } \\
\mathrm{Sd}\end{array}$ & $\begin{array}{c}9: \\
-0.8\end{array}$ & $\begin{array}{c}60 \\
2.59\end{array}$ & $\begin{array}{c}- \\
(5.7)\end{array}$ & $\begin{array}{c}21.4 \\
-17.7\end{array}$ & $\begin{array}{c}1.5 \\
0.03\end{array}$ & $\begin{array}{l}(\mathrm{MB}, 95), \\
(\mathrm{HD}, 96)\end{array}$ \\
\hline Footnotes & The & igina & stima & $D=$ & $\begin{array}{l}84 \mathrm{Mpc} \\
\mathrm{e}, D=2\end{array}$ & educe & to 2.98 & Ipc becaus & $\begin{array}{l}\text { of a new } \\
\text { aination }\end{array}$ \\
\hline
\end{tabular}

region and the lack of reliable direct estimates of distance to some of the galaxies. To judge the completeness of the present data, we collect in Table 7 some basic parameters of the galaxies around IC 342. In addition to the galaxies considered above, we include in the Table several objects whose distance and other parameters are presently known with low accuracy. In Table 7 such parameters are marked by "(:)". For 12 galaxies, ranked according to their radial velocities, the following data are presented in Table 7:

(1) — the galaxy name;

(2) - the measured radial velocity $(\mathrm{km} / \mathrm{s})$ and the radial velocity reduced to the centroid of the Local Group (Karachentsev \& Makarov 1996);
(3) - the standard angular diameter (arcmin) and apparent axial ratio;

(4) - the total blue magnitude of the galaxy and its morphological type;

(5) - the estimated absorption correction, $A_{B}$, and the galactic latitude (degrees);

(6) - the HI line width $(\mathrm{km} / \mathrm{s})$ at a level of $50 \%$ of the line peak and the log of integrated HI flux $\left(10^{-24} \cdot \mathrm{W} / \mathrm{m}^{2}\right)$;

(7) - the photometric distance modulus (mag) and the corresponding distance in $\mathrm{Mpc}$; the distances estimated via radial velocity with $H=75 \mathrm{~km} / \mathrm{s} / \mathrm{Mpc}$ are put in brackets; 

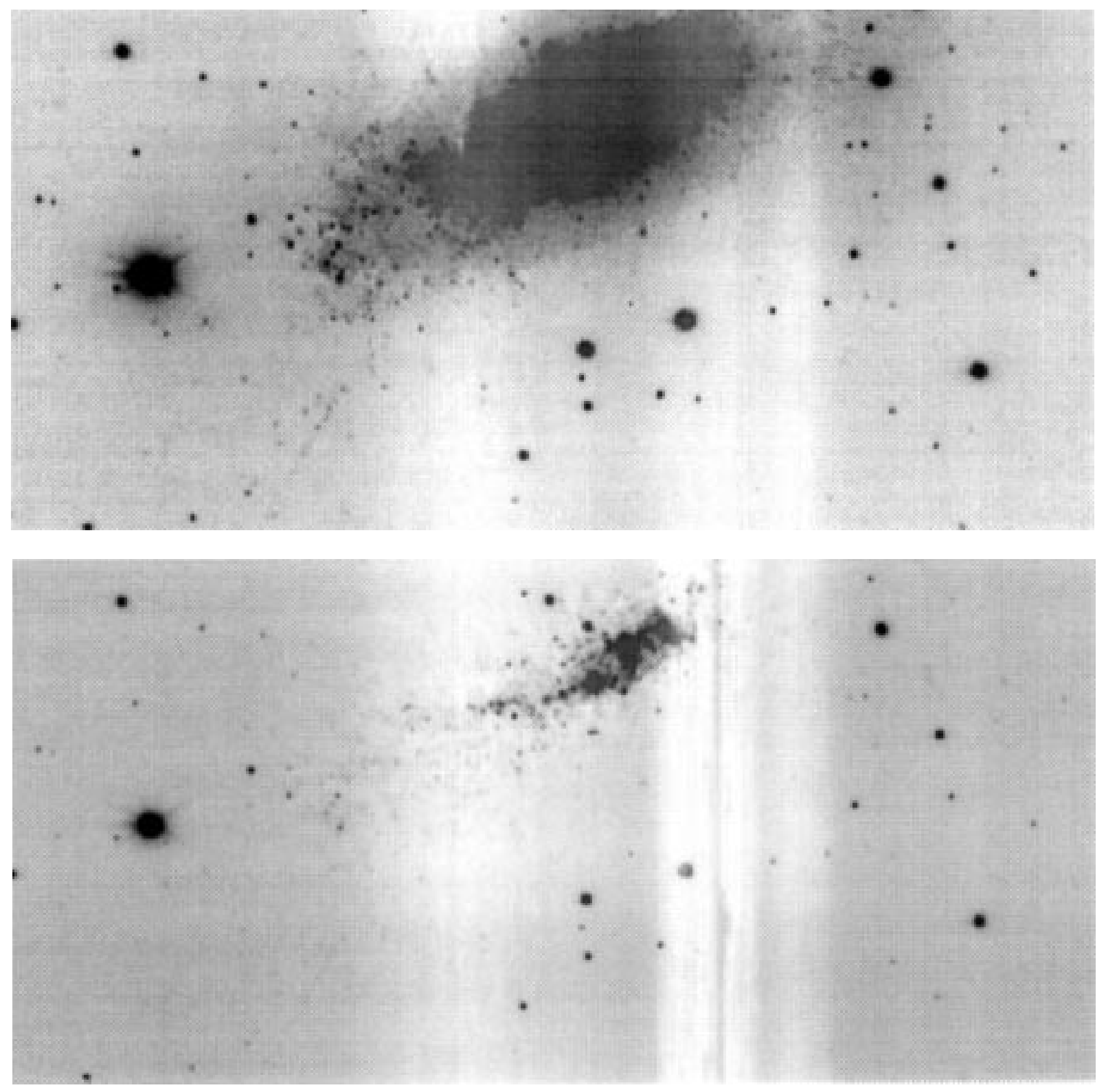

Fig. 8. NGC 1569 in the $I$ band. a) Southern half of the original frame, b) the same part after subtracting the frame smoothed with a window of $10 \times(\mathrm{FWHM})$

(8) - the standard linear diameter $(\mathrm{kpc})$ and the absolute total magnitude corrected for inclination according to RC3;

(9) - the total mass (inside the standard diameter) to total luminosity, and the total HI mass - to - the total mass (in the solar units);

(10) - notes concerning the source of the data on distance, magnitude and absorption.

The data presented in Table 7 may be useful for an analysis of the dynamical situation in the IC 342/Maffei complex of galaxies. Without going into a detailed discussion of the membership of the galaxies of the complex, we discuss here two items only. (a) In spite of possible systematic errors in the determination of the distance to the galaxies, as well as absorption estimates, the integral parameters of the galaxies considered are in a general agreement with their morphological type. A possible exception is NGC 1569 which has an abnormally low mass-toluminosity ratio. this peculiarity leads to a biased estimate of its distance $(D=0.78 \mathrm{Mpc})$ from the Tully-Fisher relation (Krismer et al. 1995).

(b) When going from normal spirals to irregular dwarfs, the standard error of distance estimate via the brightest stars remains constant, $\sigma(\mu) \simeq 0{ }^{\mathrm{m}} 4$ (Karachentsev \& Tikhonov 1994). However, the 


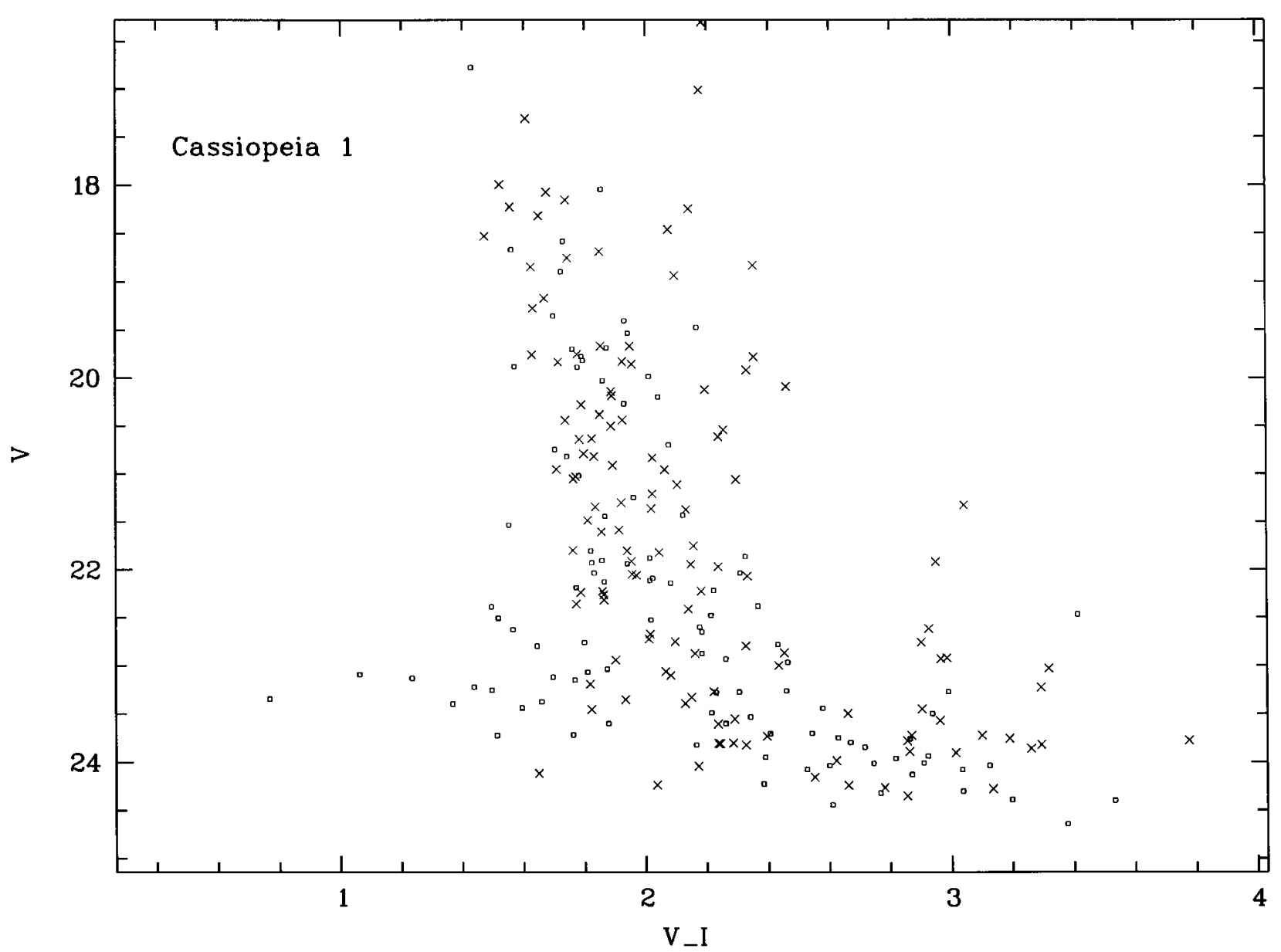

Fig. 11. $V$ vs. $(V-I)$ diagram for Cas 1 . Stars within the galaxy body are indicated by open squares the remainder of the frame stars are noted by crosses

Karachentsev I.D., Tikhonov N.A., 1993, A\&AS 100, 227 (KT, 93)

Karachentsev I.D., Tikhonov N.A., 1994, A\&A 286, 718

Karachentsev I.D., Tikhonov N.A., Georgiev Ts.B., Bilkina B.I., Sharina M.E., 1991, A\&AS 91, 503 (KTGBS, 91)

Karachentsev I.D., Tikhonov N.A., Sazonova L.N., 1994, Pis'ma v AZh 20, 104

Karachentseva V.E., Karachentsev I.D., 1997 (in preparation) Karachentseva V.E., Prugniel Ph., Vennik J., Richter G.M., Thuan T.X., 1996, A\&AS 117, 343

Karachentseva V.E., Sharina M.E., 1987, Communic. Special Astrophys. Obs. 57, 3

Kraan-Korteweg R.C., Loan A.J., Burton W.B., Lahav O., Ferguson H., Henning P.A., Lynden-Bell D., 1994, Nat 372, 77

Krismer M., Tully R.B., Gioia I.M., 1995, AJ 110, 1584

Landolt A.U., 1992, AJ 104, 340

Luppino G.A., Tonry J.L., 1993, ApJ 410, 81

McCall M.L., 1989, AJ 97, 1341

McCall M.L., Buta R.J., 1995, AJ 109, 2460 (MB, 95)
O’Connell R.W., Gallagher J.S., Hunter D.A., 1994, ApJ 433, 65

Peebles P.J.E., 1994, ApJ 429, 43

Piotto G., Capaccioli M., Bresolin F., 1992, Mem. Soc. Astr. Italian 63, 465

Sandage A.R., Tammann G.A., 1974, ApJ 191, 559; 191, 603

Spinrad H., Bahcall J., Becklin E.E., Gunn J.E., Kristian J., Neugebauer G., Sargent W.L., Smith H., 1973, ApJ 180, 351

Stetson P.B., 1987, PASP 99, 191

Tikhonov N.A., 1996, AN 317, 175 (T, 96)

Tikhonov N.A., Karachentsev I.D., 1994, Bull. Spec. Astrophys. Obs. 38, 32 (TK, 94)

Tikhonov N.A., Karachentsev I.D., Bilkina B.I., Sharina M.E., 1992, A\&A Trans. 1, 269

Tully R.B., Fisher J.R., 1977, A\&A 54, 611

Valtonen M.J., Byrd G.G., McCall M.L., Innanen K.A., 1993, AJ 105,886

Zheng J.Q., Valtonen M.J., Byrd G.G., 1991, A\&A 247, 20 